\title{
COMPOSICIÓN QUÍMICA DEL ACEITE ESENCIAL DE HOJAS DE Bejaria resinosa (Ericaceae)
}

\section{CHEMICAL COMPOSITION OF THE ESSENTIAL OIL LEAVES Bejaria resinosa (Ericaceae)}

Javier A. Matulevich Peláez, ${ }^{1,3}$ Elizabeth Gil Archila²

\section{RESUMEN}

A partir de hojas frescas de la especie vegetal Bejaria resinosa (Ericaceae) se obtuvo el aceite esencial por la técnica de hidrodestilación; la determinación de la composición química se realizó por cromatografía de gases acoplada a espectrometría de masas (CG-EM), comparación de los índices de retención, los espectros de masas y los datos reportados en la literatura. Los metabolitos secundarios identificados en el aceite esencial se cuantificaron aplicando el método de estandarización interna; además, se verificó la densidad absoluta y se determinó el índice de refracción del aceite obtenido. El aceite esencial se obtuvo con un rendimiento del $0.031 \%$ en peso; en el cual fueron identificados 26 compuestos los cuales constituyen cerca del $75 \%$ de la composición relativa total del aceite esencial, dentro de los cuales se encontraron 6 monoterpenos, 16 sesquiterpenos, y 4 hidrocarburos alifáticos, donde está presente el compuesto mayoritario, que en este caso corresponde al noneno (61.91\%). El valor obtenido para la densidad absoluta fue de 0,742 $\mathrm{g} / \mathrm{mL}$ y el índice de refracción fue de 1,4265.

Palabras clave: Bejaria resinosa, Monoterpenos, Sesquiterpenos, Aceite esencial, CG-EM.

Laboratorio de Fitoquímica GIFUJ, Departamento de Química, Facultad de Ciencias, Pontificia Universidad Javeriana, Bogotá, Colombia.

2 Laboratorio de Fitoquímica GIFUJ, Departamento de Química, Facultad de Ciencias, Pontificia Universidad Javeriana, Bogotá, Colombia.

3 Envío de correspondencia: jamatulevichp@udistrital.edu.co 
ABSTRACT

From fresh leaves of Bejaria resinous plant species (Ericaceae) essential oil by hydrodistillation technique was obtained; determining the chemical composition was performed by gas chromatography coupled to mass spectrometry (GC-MS), comparing retention index, and mass spectral data reported in the literature. Identified in the essential oil secondary metabolites were quantified using the method of internal standardization; moreover, the density was verified and the refractive index of the oil obtained was determined. The essential oil is obtained in a yield of $0.031 \%$ by weight; in which were identified 26 compounds which constitute about $75 \%$ of the relative composition of the essential oil within which 6 monoterpenes, 16 sesquiterpenes, and 4 aliphatic hydrocarbons, where the major component is present they were found in this nonene case corresponds to (61.91\%). The value obtained for the density was $0.742 \mathrm{~g} / \mathrm{mL}$ and the refractive index was 1.4265.

Key words: Bejaria resinosa, Monoterpenes, Sesquiterpenes, Essential oil, GC-MS.

\section{INTRODUCCIÓN}

La especie Bejaria resinosa perteneciente a la familia Ericaceae es un arbusto de flores vistosas de color rojo, cubiertas de una resina la cual le sirve como goma para atrapar insectos; se caracteriza por ser un arbusto de 0,5 a 2 metros de altura el cual crece entre los 2400 y 3600 msnm; las hojas son coriáceas, planas o revolutas; sus flores son bastante vistosas debido a su color y con anteras apendiculares; los frutos son capsulas globosas con exocarpo color café (Luteyn, 1995). En Colombia se distribuye en los departamentos de Antioquia, Arauca, Boyacá, Cauca, Cundinamarca, Santander y Valle; comúnmente es conocida con el nombre de pegamosco o pegajosa y es empleada localmente para tratar heridas y esguinces, como purgante y como agente preventor de ataques al corazón. De especies de Bejaria, se han realizado pocas investigaciones dentro de las cuales se encuentran estudios preliminares de la especie Bejaria glutinosa Mutis ex Linné filius en los que se encontró la presencia de triterpenos y flavonoides (Wilches, 1981) y de Bejaria resinosa donde se obtuvo una mezcla de compuestos tipo triterpeno compuesta por germanicol, $\alpha$-amirina y $\beta$-amirina y el aislamiento de lupeol, salicilato de metilo y tres flavonoides; 3,5,7,3',4' pentahidroxiflavona, 3,5-dihidroxi-6,7,8-trimetoxiflavona y 3,5,7,3',4' pentahidroxiflavanol (Matulevich, 2013).

\section{MATERIALES Y MÉTODOS}

El material vegetal fue recolectado en el municipio de Guasca, Cundinamarca (Coordenadas geográficas: $4^{\circ} 52^{\prime} 0^{\prime \prime} \mathrm{N}, 73^{\circ} 53^{\prime} 0^{\prime \prime} \mathrm{W}$ ) en el mes de mayo de 2011. Una muestra testigo fue enviada al Herbario Nacional de Colombia para su determinación taxonómica la cual fue clasificada como Bejaria resinosa Mutis ex L. f. bajo el número de colección COL 565500. El aceite esencial fue obtenido a partir de hojas frescas (950 g) por hidrodestilación, obteniéndose 0,5 ml de aceite. La determinación de la composición química relativa del aceite esencial se realizó por CG-EM en un equipo SHIMADZU 
QP2010 plus, empleando una columna capilar de sílice fundida, HP-5MS (J \& W Scientific, Folsom, CA, EE.UU.) de $60 \mathrm{~m} \times 0,25 \mathrm{~mm} \times 0,25 \mu \mathrm{m}$, con fase estacionaria $5 \%$ fenilpolimetilsiloxano. La programación de temperatura del horno fue de $45^{\circ} \mathrm{C}$ (5 min) @ 5 ${ }^{\circ} \mathrm{C} / \mathrm{min}$, hasta $60^{\circ} \mathrm{C}(1 \mathrm{~min}) @ 30^{\circ} \mathrm{C} / \mathrm{min}$, hasta $130^{\circ} \mathrm{C}$ (0 min) @ $4^{\circ} \mathrm{C} / \mathrm{min}$, hasta $190^{\circ} \mathrm{C}$ (2 min) @ $40^{\circ} \mathrm{C} / \mathrm{min}$, hasta $285^{\circ} \mathrm{C}$ (0 min). Los espectros de masas se obtuvieron por ionización electrónica (IE) de energía de 70 eV. Las temperaturas de la cámara de ionización y de la línea de transferencia fueron de 230 y 325 de los picos cromatográficos de cada uno de los componentes de la muestra se compararon con el área reportada para el estándar interno. Además, se verifico la densidad absoluta del aceite esencial determinando el peso de $100 \mu \mathrm{L}$ del producto obtenido en un matraz previamente seco y pesado a una temperatura de trabajo de $18 \pm 2^{\circ} \mathrm{C}$ y se determinó el índice de refracción en un refractómetro Carl Zeius Jena, el cual fue ajustado con agua destilada verificando su refracción en 1.3330, siguiendo las instrucciones del fabricante.

De especies de Bejaria, se han realizado pocas investigaciones dentro de las cuales se encuentran estudios preliminares de la especie Bejaria glutinosa Mutis ex Linné filius en los que se encontró la presencia de triterpenos y flavonoides.

${ }^{\circ} \mathrm{C}$, respectivamente. El gas de arrastre utilizado fue helio (grado 5.0), con flujo constante de 1,2 mL/min. Los índices IR se calcularon teniendo en cuenta los tiempos de retención de una serie homologa de patrones de hidrocarburos desde $\mathrm{C}_{7}$ hasta $\mathrm{C}_{24^{\prime}}$ analizados por CG-EM bajo las mismas condiciones que el aceite esencial.

Los metabolitos secundarios identificados en el aceite esencial se cuantificaron aplicando el método de estandarización interna empelando un detector EM; para ello se empleó n-tetradecano (Merck). La muestra para análisis se preparó tomando una alícuota de $50 \mu \mathrm{L}$ del aceite esencial obtenido, se le agrego 1,0 $\mu \mathrm{L}$ de patrón interno (n-tetradecano), y se completó a $1 \mathrm{~mL}$ con diclorometano. Las áreas

\section{RESULTADOS Y DISCUSIÓN}

El aceite esencial de las hojas de Bejaria resinosa se obtuvo con un rendimiento del $0.031 \%$; el valor obtenido para la densidad absoluta fue de 0,742 g/ $\mathrm{ml}$ y el índice de refracción fue de 1,4265. La identificación de los componentes presentes en el aceite esencial se realizó comparando los índices de retención y los espectros de masas con los datos reportados en la literatura (Adams, 1995; Goodner, 2007). Se identificaron 26 compuestos, entre ellos monoterpenos, sesquiterpenos e hidrocarburos alifáticos; estos últimos constituyen cerca del $67 \%$ de la composición relativa total del aceite esencial. En la tabla 1 se presentan los compuestos identificados por 
Tabla 1. Composición química relativa del aceite esencial obtenido en la columna RTX-5.

\begin{tabular}{|c|c|c|c|c|c|c|c|}
\hline $\begin{array}{l}\text { No. } \\
\text { Señal }\end{array}$ & tRX & IR cal & $\%$ & $\begin{array}{l}\text { Concentración } \\
(\mu \mathrm{g} / \mathrm{mL})\end{array}$ & Nombre & * & IR Ref \\
\hline 1 & 13,367 & 810 & 3,40 & 229,9 & Octeno & HA & 807 \\
\hline 2 & 18,153 & 921 & 61,91 & 4187,7 & Noneno & $\mathrm{HA}$ & 906 \\
\hline 3 & 19,533 & 922 & 0,88 & 59,5 & 1,3-nonadieno & HA & 914 \\
\hline 4 & 19,987 & 939 & 0,36 & 24,3 & $\alpha$-pineno & $M$ & 939 \\
\hline 5 & 22,307 & 983 & 1,99 & 134,6 & $\beta$-pineno & $\mathrm{M}$ & 980 \\
\hline 6 & 24,167 & 1036 & 1,42 & 96,0 & Limoneno & $\mathrm{M}$ & 1031 \\
\hline 7 & 24,267 & 1039 & 0,64 & 43,2 & $\beta$-felandreno & M & 1031 \\
\hline 8 & 24,747 & 1041 & 2,20 & 148,8 & orto-Cimeno & M & 1022 \\
\hline 9 & 26,593 & 1110 & 1,22 & 82,5 & Undeceno & $\mathrm{HA}$ & 1100 \\
\hline 10 & 26,913 & 1104 & 0,34 & 22,9 & Linalol & $\mathrm{M}$ & 1098 \\
\hline 11 & 37,573 & 1377 & 0,34 & 22,9 & $\alpha$-Copaeno & $\mathrm{S}$ & 1376 \\
\hline 12 & 38,273 & 1418 & 0,19 & 12,8 & $\beta$ panasinseno & S & 1411 \\
\hline 13 & 39,273 & 1423 & 0,42 & 28,4 & $\beta$-Cariofileno & $\mathrm{S}$ & 1418 \\
\hline 14 & 39,907 & 1464 & 0,27 & 18,2 & $\beta$-farneseno & $\mathrm{S}$ & 1458 \\
\hline 15 & 41,113 & 1480 & 0,43 & 29,0 & Y-himachaleno & S & 1476 \\
\hline 16 & 41,400 & 1525 & 0,40 & 27,0 & $\beta$-sesquifelandreno & S & 1524 \\
\hline 17 & 41,560 & 1529 & 0,40 & 27,0 & Calameneno & S & 1532 \\
\hline 18 & 41,880 & 1538 & 1,62 & 109,5 & $\delta$-cadineno & S & 1538 \\
\hline 19 & 42,040 & 1543 & 0,23 & 15,5 & 3,7-(11)-dienoselina & S & 1542 \\
\hline 20 & 42,667 & 1560 & 0,53 & 35,8 & $\beta$-Calacoreno & S & 1563 \\
\hline 21 & 43,280 & 1565 & 0,29 & 19,6 & a-Calacoreno & S & 1550 \\
\hline 22 & 45,353 & 1581 & 0,27 & 18,2 & Oxido de cariofileno & $\mathrm{s}$ & 1581 \\
\hline 23 & 43,773 & 1593 & 0,24 & 16,2 & Epóxido de humuleno & S & 1606 \\
\hline 24 & 46,773 & 1652 & 0,44 & 29,7 & Cubenol & S & 1642 \\
\hline 25 & 47,087 & 1658 & 0,22 & 14,8 & $\beta$-eudesmol & S & 1649 \\
\hline 26 & 48,053 & 1653 & 0,80 & 54,1 & $\alpha$-eudesmol & S & 1652 \\
\hline
\end{tabular}


comparación con índices de retención y con la librería NIST 08; se reportaron los compuestos que por comparación presentaban más de un $90 \%$ de coincidencia con el espectro de la librería, también se presentan las cantidades relativas que corresponden al porcentaje de abundancia de cada componente dentro del aceite esencial y la cuantificación por el método de estandarización interna.

En el aceite esencial fueron identificados 26 compuestos (81\%); 6 monoterpenos (M), 16 sesquiterpenos (S), y 4 hidrocarburos alifáticos (HA), entre los que se encuentra el compuesto mayoritario, que en este caso corresponde al noneno (61.91\%). Los sesquiterpenos encontrados representan el 7,09\% de la composición del aceite esencial, en los cuales los componentes mayoritarios encontrados fueron: $\delta$-cadineno (1,62\%), a-eudesmol $(0,80 \%)$ y $\beta$-Calacoreno (0,53\%). En cuanto a los monoterpenos representan tan solo el 6,95\% donde el constituyente en mayor porcentaje es el o-Cimeno (2,2\%) seguido de $\beta$-pineno (1,99\%) y limoneno $(1,42 \%)$.

Teniendo en cuenta que el rendimiento fue inferior al $0,1 \%$ se puede considerar a Bejaria resinosa como una especie vegetal pobre en aceites esenciales, lo cual está de acuerdo con los componentes volátiles determinados, ya que especies con bajos rendimientos se caracterizan por producir cantidades considerables de compuestos derivados de ácidos grasos, esteres de ácidos grasos y derivados carotenoides; a diferencia de especies con alto rendimiento las cuales se caracterizan por producir gran cantidad de monoterpenos y fenilpropanoides (Radulovic et al; 2010).

Otros reportes en la literatura (Radulovic et al; 2010) muestran los componentes de otras especies pertenecientes a la familia Ericaceae; como es el caso de las especies vegetales Arctostaphylos uvaursi y Vaccinium vitis-idaea donde a partir de las hojas fueron extraídos los aceites esenciales encontrando predominancia de terpenos, ácidos grasos, derivados de carotenoides y además a-terpineol y linalol los cuales son considerados taxones químicos de los géneros Arctostaphylos y Vaccinium (Radulovic et al; 2010).

Al realizar la comparación de los componentes identificados en el aceite esencial de Bejaria resinosa con los de las especies Arctostaphylos uva-ursi y Vaccinium vitis-idaea se encuentran como compuestos comunes para las tres especies el linalol, $\beta$-Cariofileno, $\beta$-farneseno, humuleno, 3,7-(11)-dienoselina y $\beta$-eudesmol y además un porcentaje de sesquiterpenos muy cercano entre ellos. El presente estudio es un aporte a las investigaciones fitoquímicas de la familia Ericaceae en Colombia y en particular del género Bejaria, ya que éste es el primer reporte en cuanto a la constitución de metabolitos volátiles de un género que cuenta con escasos estudios químicos.

\section{AGRADECIMIENTOS}

Los autores expresan sus agradecimientos a la Pontificia Universidad Javeriana y a la Universidad Distrital Francisco José de Caldas 


\section{REFERENCIAS}

1. Adams, R. (1995). Identification of essential oil components by gas chromatography / mass spectroscopy. Allured Publishing Corporation. USA, $468 \mathrm{p}$.

2. Babushok, V., \& Zenkevich, I. (2009). Retention Indices for most frequently reported essential oil compounds in GC. Chromatographia, 69: 257-269.

3. Clemants, S. (1991). Two New Species of Bejaria (Ericaceae) from South America. Brittonia, 43: 171-177.

4. Goodner, K. (2008). Practical retention index models of OV-101, DB-1, DB-5, and DB-Wax for flavor and fragance compounds. LWT Food Science and Technology, 41: 951-958.

5. Luteyn, J. (1995). Ericaceae. Organization for Flora Neotrópica. New York Botanical Garden. New York, 1034 p.
6. Matulevich, J. (2013). Estudio fitoquímico de hojas, flores y frutos de Bejaria resinosa Mutis ex Linné filius (Ericaceae) y evaluación de su actividad antiinflamatoria. Trabajo de grado, Programa de Maestría en Ciencias Biológicas, Facultad de Ciencias, Pontificia Universidad Javeriana. Bogotá, 93 p.

7. Radulovic, N., Blagojevic, P., \& Palic, R. (2010). Comparative study of the leaf volatiles of Arctostaphylos uva-ursi (L) Spreng and Vaccinium vitis-idaea L. (Ericaceae). Molecules, 15: 6168-6185.

8. Wilches, F. (1981). Contribución al estudio fitoquímico de Befaria glutinosa y sus posibles efectos fisiológicos. Trabajo de grado, Programa de Maestría en Ciencias Biológicas, Facultad de Ciencias, Pontificia Universidad Javeriana. Bogotá, 125 p. 